$63^{\text {ème }}$ Congrès de la SFCO, 02018 (2015)

DOI:10.1051/sfco/20156302018

(C) Owned by the authors, published by EDP Sciences, 2015

\title{
COMMUNICATION
}

\section{Compression du nerf alvéolaire inférieur d'origine endodontique, intérêt de la piézochirurgie, présentation de deux cas}

\author{
Cros A, Cousty S, Labadie M-P, L'Homme A, Courtois B \\ Département de Chirurgie Orale Toulouse
}

Le pronostic de guérison des lésions nerveuses d'origine endodontique par compression est excellent si l'élimination du facteur compressif et potentiellement neurotoxique, c'est-à-dire le corps étranger est rapide. Cette exérèse permet aussi de supprimer la réaction inflammatoire locale liée à la présence de ce corps étranger et de rétablir un environnement propre à une cicatrisation nerveuse ${ }^{1}$.

L'intervention chirurgicale permet de décomprimer la fibre nerveuse mais également de visualiser directement les dommages sur la fibre. En effet les examens cliniques et radiologiques ne permettent que un diagnostic présumé de type d'atteinte de la fibre nerveuse (compression seule, rupture de l'épinèvre avec diffusion du ciment endodontique au sein de la fibre, ...) selon la classification de Sunderland et Seddon. Seule l'exploration chirurgicale permet le diagnostic et donc le pronostic de récupération de la sensibilité.

De nombreuses voies d'abord ont été proposées pour accéder au nerf alvéolaire inférieur. Une approche extra-orale ou intra-orale, des abords par ostéotomie sagittale, par décortication vestibulaire ou par l'alvéole après avulsion de la dent causale. Quelle que soit la voie d'abord choisie, l'utilisation d'une instrumentation la moins iatrogène possible est nécessaire pour une intervention sur un tissus aussi sensible qu'une fibre nerveuse.

La chirurgie ultra sonore est maintenant largement utilisée dans de nombreux domaines chirurgicaux. Elle permet une approche chirurgicale économe en terme de tissus et sécuritaire pour les tissus mous lors de la réalisation des ostéotomies, du curetage osseux et de l'éviction du corps étranger au contact du nerf. Elle permet également une bonne visibilité du site opératoire, indispensable à un examen du sitechirurgical, en réduisant le saignement par phénomène de cavitation. La guérison osseuse bénéficie également de cette chirurgie moins traumatique ${ }^{2}$.

La récupération d'une lésion nerveuse de compression est le plus souvent complète en cas d'intervention chirurgicale. Dans les cas où les patients ont refusé toute chirurgie, la récupération est lente et souvent incomplète. Une paresthésie labio-mentonnière, d'autres troubles nerveux locaux peuvent persister plusieurs années ${ }^{3}$.

Deux cas cliniques de décompression du nerf alvéolaire inférieur sont présentés, pris en charge dans le service de chirurgieorale de Rangueil. Deux patientes ont été traitées par corticotomie vestibulaire sous anesthésie locale. La première patiente souffrait d'hyperesthésies sur le site de la dent traitée depuis deux semaines. La deuxième patiente présentait des symptômes des hyperesthésies locales associées à des paresthésies labiomentonnières d'apparition progressive 1 mois après le traitement endodontique. Le recouvrement de la sensibilité normale s'est fait directement après la chirurgie pour la patiente $n^{\circ} 1$ et plusieurs mois après pour la patiente $n^{\circ} 2$, mais dans les 2 cas la chirurgie a montré un succès immédiat quant à la résolution des douleurs neurologiques.

This is an Open Access article distributed under the terms of the Creative Commons Attribution License 4.0, which permits unrestricted use, distribution, and reproduction in any medium, provided the original work is properly cited. 
1- Scolozzi et Lombardi (2004)

2. Pavliková, Foltá et col (2011)

3 - González-Martín et Torres-Lagares et col (2010)

Nom et adresse du conférencier

\section{Alice CROS}

Faculté d'odontologie de Toulouse

3 chemin des maraîchers

31059 Toulouse Cedex 9 (France)

drcrosalice@gmail.com 